\title{
APPROXIMATE SIMILARITY AND ALMOST PERIODIC MATRICES ${ }^{1}$
}

\author{
JAMES C. LILLO
}

1. Introduction and notation. We denote by $M_{n}$ the set of all matrices which are bounded continuous real valued functions of $t$ for $-\infty<t<\infty . F^{n}$ denotes the space of all elements of $M_{n}$ with almost periodic entries and the topology defined by the uniform norm (i.e., $\mathfrak{R}[A(t)]=1$. u.b. - $\left.<<<\infty \infty\left[\sum_{i, j=1}^{n}\left|a_{i j}(t)\right|\right]\right)$. A matrix $A(t) \in M_{n}$ is said to be approximately similar to $B(t) \in M_{n}$, written $A(t) \approx B(t)$, if given any $\epsilon>0$ there exists $P(\epsilon, t)$ such that $P(\epsilon, t), P^{-1}(\epsilon, t), \dot{P}(\epsilon, t) \in M_{n}$ and $\mathfrak{R}\left[P^{-1}(-\dot{P}+A(t) P)-B(t)\right]<\epsilon$.

The above concept of approximate similarity was introduced by the author to study questions of stability [1] and perturbations of almost periodic solutions [2], of nonlinear differential equations. In both cases, elements of $F^{n}$ which are approximately similar to constant matrices were of considerable importance. One might expect that every element of $F^{n}$ is approximately similar to some constant matrix. Unfortunately the author has not yet succeeded in establishing this result. Here we prove a much weaker result. Let $G^{n}$ denote the set of all elements $B(t) \in F^{n}$ for which there exists a real constant matrix $A$, whose eigenvalues are all real and distinct such that $B(t)$ is approximately similar to $A$. Theorem 2.3 tells us that the set $G^{n}$ is an open set in $F^{n}$.

The work of Perron assures us that systems of the form:

$$
\dot{x}=B(t) x, \quad B(t) \in F^{n},
$$

may be reduced by a transformation $T(t)$ to a system of the form

$$
\dot{y}=A(t) y,
$$

where $A(t) \in M_{n}$ and is upper triangular, and $T(t), \dot{T}(t), T^{-1}(t) \in M_{n}$. Unfortunately, the Perron transformation, $T(t)$, is not constructible in the sense that it depends upon a fundamental system of solutions of (1.1). A natural question, however, is the existence of a Perron transformation $T(t)$, with $T(t), T^{-1}(t), \dot{T}(t) \in F^{n}$. In this case $A(t)$ in system (1.2) belongs to $F^{n}$ in addition to being upper triangular. For such systems a number of results are available [3]. In Theorem 2.1 we

Presented to the Society, January 27, 1961; received by the editors May 26, 1960.

1 This research was partially supported by the United States Air Force through the Air Force Office of Scientific Research of the Air Research and Development Command, under Contract Number AF 49(638)-382. Reproduction in whole or in part is permitted for any purpose of the United States Government. 
show that if $B(t) \in G^{n}$ then system (1.1) possesses at least one almost periodic Perron transformation. In $\$ 3$ we show by means of an example that the set of $B(t) \in F^{n}$ for. which there exists at least one almost periodic Perron transformation is not open in $F^{n}$.

2. Almost periodic Perron transformations. In preparation for Theorems 2.1 and 2.3 we now establish four lemmas. In order to facilitate the statements and proofs we introduce the following definitions. A matrix $B(t) \in M_{n}$ is said to satisfy condition $\mathrm{I}$ if there exist real constants $\lambda_{1}, \cdots, \lambda_{n}$, with $\mu=\min _{i \neq j}\left(\left|\lambda_{i}-\lambda_{j}\right| / 4\right) \neq 0$, and there exists an $M>0$ such that the system $\dot{x}=B(t) x$ possesses $n$ solutions $\rho_{1}(t), \cdots, \rho_{n}(t)$, which satisfy the inequalities:

$$
\begin{aligned}
(1 / M) & \exp \left[\lambda_{j}\left(t-t_{0}\right)-\mu\left|t-t_{0}\right|\right] \leqq\left\|\rho_{j}(t)\right\| /\left\|\rho_{j}\left(t_{0}\right)\right\| \\
& \leqq M \exp \left[\lambda_{j}\left(t-t_{0}\right)+\mu\left|t-t_{0}\right|\right], j=1, \cdots, n,-\infty<t<\infty .
\end{aligned}
$$

If $B(t)$ satisfies condition I, then any set of solutions $\rho_{1}(t), \cdots, \rho_{n}(t)$ which satisfy the above inequalities will be called a distinguished set. Finally if $A(t) \in M_{n}$ then $[A(t)]^{j}$ and $[A(t)]_{l}$ denote the $j$ th column and $l$ th row of $A(t)$, respectively.

LeMma 2.1. If $A=\operatorname{diag}\left(\lambda_{1}, \cdots, \lambda_{n}\right), \lambda_{i}$ real and

$$
\mu=\min _{i \neq j}\left(\left|\lambda_{i}-\lambda_{j}\right| / 4\right) \neq 0,
$$

then there exists an open set of $M_{n}$ containing $A$ such that every element in this set satisfies condition I.

Proof. We are interested in systems of the form

$$
\dot{x}=[A+B(t)] x
$$

where $B(t) \in M_{n}, \mathfrak{N}[B]$ is small, and $A=\operatorname{diag}\left(\lambda_{1}, \cdots, \lambda_{n}\right)$. We may assume without loss of generality that $\lambda_{i}<\lambda_{i+1}$, for $i=1,2, \cdots, n-1$. For systems of this form it is possible to show [2] that there exist $n$ independent solutions $\rho_{j}(t)$ which satisfy the following inequalities:

$$
\begin{array}{r}
(1 / 8) \exp \left(\lambda_{j} t-\mu|t|\right) \leqq\left\|\rho_{j}(t)\right\| /\left\|\rho_{j}(0)\right\| \leqq 8 \exp \left(\lambda_{j} t+\mu|t|\right) \\
j=1,2, \cdots, n .
\end{array}
$$

In the above inequalities $\mu$ may be made arbitrarily small by taking $\mathfrak{N}[B]$ sufficiently small. These are the desired inequalities except that $t_{0}$ is restricted to $t_{0}=0$.

We now remove that restriction. We first observe that in obtaining the inequalities (2.2) the only property of $B(t)$ that is used is its norm (i.e., $\mathfrak{N}[B]$ ). Thus if we consider the system 


$$
\dot{x}=\left[A+B\left(t+t_{0}\right)\right] x,
$$

we obtain a set of $n$ solutions $\rho_{j}^{*}(t)$ satisfying inequalities of the form (2.2). However, up to a constant multiple, the inequalities (2.2) completely determine the $\rho_{j}(t)$. Thus there exist nonzero constants $k_{j}$ such that $\rho_{j}\left(t+t_{0}\right)=k_{j} \rho_{j}^{*}(t)$ and then from the inequalities for $\rho_{j}^{*}(t)$ we obtain:

$$
(1 / 8) \exp \left[\lambda_{j}\left(t-t_{0}\right)-\mu\left|t-t_{0}\right|\right] \leqq\left\|\rho_{j}(t)\right\| /\left\|\rho_{j}\left(t_{0}\right)\right\|
$$

$$
\leqq 8 \exp \left[\lambda_{j}\left(t-t_{0}\right)+\mu\left|t-t_{0}\right|\right]
$$

where $t$ and $t_{0}$ are arbitrary. This completes the proof of Lemma 2.1.

Lemma 2.2. Let $A(t) \in F^{n}$. Then if $A(t)$ satisfies condition I with constants $\lambda_{1}, \lambda_{2}, \cdots, \lambda_{n}, \mu$, and $M$, every $A^{*}(t)$ in the closed hull of $A(t)$ satisfies condition I with constants $\lambda_{1}, \cdots, \lambda_{n}, \mu$, and $M$.

Proof. If $A^{*}(t)$ belongs to the hull of $A(t)$ then there exists a sequence $\left\{\tau_{i}\right\}$ such that $A^{*}(t)$ is the uniform limit of $A\left(t+\tau_{i}\right)$ as $i$ tends to $\infty$. Let $\rho_{j}(t), j=1, \cdots, n$, be a distinguished set of solutions for the system $\dot{x}=A(t) x$. Clearly there exist $n$ unit vectors $p_{j}$ and a subsequence $\left\{\tau_{i}^{*}\right\}$ of $\left\{\tau_{i}\right\}$ such that $\lim _{i \rightarrow \infty}\left\{\rho_{j}\left(\tau_{i}^{*}\right) /\left\|\rho_{j}\left(\tau_{i}^{*}\right)\right\|\right\}=p_{j}$. Now denote by $\rho_{j}^{*}(t)$ the solutions of $\dot{x}=A^{*}(t) x$ which satisfy the initial conditions $\rho_{j}^{*}(0)=p_{j}$. Then it is clear that for $t$ in any finite interval the solutions $\rho_{j}\left(t+\tau_{i}^{*}\right) /\left\|\rho_{j}\left(\tau_{i}^{*}\right)\right\|$ converge uniformly to the $\rho_{j}^{*}(t)$. Thus the $\rho_{j}^{*}(t), j=1,2, \cdots, n$, satisfy the inequalities of condition I and we have our desired result.

Before proceeding to Lemmas 2.3 and 2.4 we introduce some further notation. We define $L^{n}$ as the set of elements belonging to $F^{n}$ which are upper triangular and whose diagonal terms all possess distinct mean values (i.e., if $B(t) \in L^{n}$ then mean value of $b_{i i}(t) \neq$ mean value of $b_{j j}(t)$ for $\left.i \neq j\right)$. If $\rho_{1}(t), \cdots, \rho_{n}(t)$ are $n$ independent solutions of $\dot{x}=A(t) x$ we shall denote the associated Perron transformation matrix, which is obtained by applying the Gram-Schmidt process to the column vectors $\rho_{i}(t)$, by G.S. $\left[\rho_{1}(t), \cdots, \rho_{n}(t)\right]$. For a further discussion of this procedure the reader is referred to a paper by Reid [5]. We now note that if $\sigma_{i}(t)=k_{i} \rho_{i}(t)$ where $k_{i} \neq 0, i=1,2, \cdots, n$, then

$$
\begin{aligned}
& \text { G.S. }\left[\sigma_{1}(t), \cdots, \sigma_{n}(t)\right]=\text { G.S. }\left[k_{1} \rho_{1}(t), \cdots, k_{n} \rho_{n}(t)\right] \\
& \quad=\text { G.S. }\left[\rho_{1}(t), \cdots, \rho_{n}(t)\right] \times \operatorname{diag}\left[\operatorname{sgn}\left(k_{1}\right) \cdot 1, \cdots, \operatorname{sgn}\left(k_{n}\right) \cdot 1\right] .
\end{aligned}
$$

Now if $B(t)$ satisfies condition I and $\rho_{1}(t), \cdots, \rho_{n}(t)$, and $\sigma_{1}(t), \cdots$, $\sigma_{n}(t)$ are two sets of distinguished solutions then there exists $k_{i} \neq 0$ 
such that $\sigma_{i}(t)=k_{i} \rho_{i}(t), i=1,2, \cdots, n$. Thus

$\|$ G.S. $\left[\sigma_{1}(t), \cdots, \sigma_{n}(t)\right]-$ G.S. $\left[\rho_{1}(t), \cdots, \rho_{n}(t)\right] \|$

$=\|$ G.S. $\left[\rho_{1}(t), \cdots, \rho_{n}(t)\right] \times\left(\mathrm{I}-\operatorname{diag}\left[\operatorname{sgn}\left(k_{1}\right) \cdot 1, \cdots, \operatorname{sgn}\left(k_{n}\right) \cdot 1\right]\right) \|=2 n k$

where $k$ is the number of $k_{i}$ which are negative. In particular, either

$$
\text { G.S. }\left[\sigma_{1}(t), \cdots, \sigma_{n}(t)\right] \equiv \text { G.S. }\left[\rho_{1}(t), \cdots, \rho_{n}(t)\right]
$$

or else

$$
\| \text { G.S. }\left[\sigma_{1}(t), \cdots, \sigma_{n}(t)\right]-\text { G.S. }\left[\rho_{1}(t), \cdots, \rho_{n}(t)\right] \| \geqq 2 n
$$

for all $t$. Finally we note that if $A(t) \in F^{n}$ and satisfies condition I and if $A^{*}(t)$ belongs to the hull of $A(t)$ then if we consider the distinguished set $\rho_{1}(t), \cdots, \rho_{n}(t)$ for the system $\dot{x}=A(t) x$ there exists a sequence $\left\{\tau_{i}\right\}$ such that $\left\{A\left(t+\tau_{i}\right)\right\}$ converges uniformly to $A^{*}(t)$ and the $\rho_{j}\left(t+\tau_{i}\right) /\left\|\rho_{j}\left(\tau_{i}\right)\right\|$ converges to a distinguished set $\rho_{j}^{*}(t)$ for the system $\dot{x}=A^{*}(t) x$. Furthermore for any value of $t$,

$$
\text { G.S. }\left[\rho_{1}^{*}(t), \cdots, \rho_{n}^{*}(t)\right]=\lim _{i \rightarrow \infty} \text { G.S. }\left[\rho_{1}\left(t+\tau_{i}\right), \cdots, \rho_{n}\left(t+\tau_{i}\right)\right]
$$

and in fact this convergence is uniform for $t$ in compact intervals. We now consider Lemma 2.3.

LEMma 2.3. If $B(t) \in F^{n}$ and satisfies condition I with constants $\lambda_{1}, \cdots, \lambda_{n}$ then $B(t)$ is kinematically similar [4] (by means of an almost periodic transformation) to a matrix $C(t) \in L^{n}$. The mean values $\lambda_{i}^{*}$ of $c_{i i}(t)$ satisfy the inequalities $\lambda_{i}-\mu \leqq \lambda_{i}^{*} \leqq \lambda_{i}+\mu$.

Proof. Since $B(t)$ satisfies condition I there exists a distinguished set $\rho_{1}(t), \cdots, \rho_{n}(t)$. We assert that G.S. $\left[\rho_{1}(t), \cdots, \rho_{n}(t)\right]$ is almost periodic. We show, in fact, that the family of matrices

$$
\text { G.S. }\left[\rho_{1}(t+\tau), \cdots, \rho_{n}(t+\tau)\right] \text {, where }-\infty<\tau<\infty \text {, }
$$

is a normal family. It is clearly a uniformly bounded and equicontinuous family. Thus we need only show that for any sequence $\left\{\tau_{i}\right\}$ there exists a subsequence $\left\{\tau_{i}^{*}\right\}$ such that G.S. $\left[\rho_{1}\left(t+\tau_{i}^{*}\right), \cdots, \rho_{n}\left(t+\tau_{i}^{*}\right)\right]$ converges uniformly as $i \rightarrow \infty$. Since $B(t)$ is almost periodic, by Lemma 2.2 and the remarks which follow it, there exists a subsequence $\left\{\tau_{i}^{*}\right\}$ such that $B\left(t+\tau_{i}^{*}\right)$ converges uniformly to $A^{*}(t)$ and the $\rho_{j}\left(t+\tau_{i}^{*}\right) /\left\|\rho_{j}\left(\tau_{i}^{*}\right)\right\|$ converge to a distinguished set $\sigma_{j}(t)$ for the system $\dot{x}=A^{*}(t) x$. We now assert that G.S. $\left[\rho_{1}\left(t+\tau_{i}^{*}\right), \cdots, \rho_{n}\left(t+\tau_{i}^{*}\right)\right]$ converges uniformly to G.S. $\left[\sigma_{1}(t), \cdots, \sigma_{n}(t)\right]$. If not, there exists an $\epsilon, 1 / 2>\epsilon>0$, a sequence $\left\{t_{i}\right\}$ and an infinite set of positive integers $\{h(i)\}$ such that 


$$
\begin{aligned}
1 / 2> & \| \text { G.S. }\left[\rho_{1}\left(t_{h(i)}+\tau_{h(i)}^{*}\right), \cdots, \rho_{n}\left(t_{h(i)}+\tau_{h(i)}^{*}\right)\right] \\
& - \text { G.S. }\left[\sigma_{1}\left(t_{h(i)}\right), \cdots, \sigma_{n}\left(t_{h(i)}\right)\right] \|>\epsilon .
\end{aligned}
$$

In addition we may assume that the sequence $\{h(i)\}$ has been chosen in such a way that the limits:

$$
\lim _{i \rightarrow \infty} A^{*}\left(t+t_{h(i)}\right)=\lim _{i \rightarrow \infty} B\left(t+t_{h(i)}+\tau_{h(i)}^{*}\right)=B^{*}(t)
$$

exist uniformly in $t$ and finally that the $\sigma_{j}\left(t+t_{h(i)}\right) /\left\|\sigma_{j}\left(t_{h(i)}\right)\right\|$ and $\rho_{j}\left(t+t_{h(i)}+\tau_{h(i)}^{*}\right) /\left\|\rho_{j}\left(t_{h(i)}+\tau_{h(i)}^{*}\right)\right\|$ converge to distinguished sets for $B^{*}(t)$ which we denote by $\sigma_{1}^{*}(t), \cdots, \sigma_{n}^{*}(t)$ and $\rho_{1}^{*}(t), \cdots, \rho_{n}^{*}(t)$ respectively. But this means that

$$
\text { G.S. }\left[\rho_{1}\left(t+t_{h(i)}+\tau_{h(i)}^{*}\right), \cdots, \rho_{n}\left(t+t_{h(i)}+\tau_{h(i)}^{*}\right)\right]
$$

converges to G.S. $\left[\rho_{1}^{*}(t), \cdots, \rho_{n}^{*}(t)\right]$ uniformly for $t$ in any compact interval. However, setting $t=0$, we obtain the inequality $1 / 2$ $>\|$ G.S. $\left[\sigma_{1}^{*}(0), \cdots, \sigma_{n}^{*}(0)\right]-$ G.S. $\left[\rho_{1}^{*}(0), \cdots, \rho_{n}^{*}(0)\right] \|>\epsilon$ which is impossible since $\sigma_{1}^{*}(t), \cdots, \sigma_{n}^{*}(t)$ and $\rho_{1}^{*}(t), \cdots, \rho_{n}^{*}(t)$ are both distinguished sets for $B^{*}(t)$. This then proves that for any distinguished set the associated Perron transformation is almost periodic. Since its derivative is uniformly bounded and uniformly continuous it is likewise almost periodic. Thus if $\rho_{1}(t), \cdots, \rho_{n}(t)$ is a distinguished set for $B(t) \in F^{n}$ the transformation G.S. $\left[\rho_{1}(t), \cdots, \rho_{n}(t)\right]$ will reduce the system $\dot{x}=B(t) x$ to one of the form $\dot{y}=C(t) y$ where $C(t) \in L^{n}$. The statement concerning the mean values of $c_{i i}(t)$ now follows from known results [1].

LEMMA 2.4. If $B(t) \in F^{n}$ is kinematically similar to an element $C(t) \in L^{n}$ where $\lambda_{i}^{*}$ is the mean value of $c_{i i}(t)$ then $B(t)$ is approximately similar to $\operatorname{diag}\left(\lambda_{1}^{*}, \cdots, \lambda_{n}^{*}\right)$.

Proof. Since approximate similarity is a transitive relationship, the desired result follows immediately from the fact that $B(t)$ is approximately similar to $\operatorname{diag}\left[c_{11}(t), \cdots, c_{n n}(t)\right][1]$ and that $\operatorname{diag}\left[c_{11}(t), \cdots, c_{n n}(t)\right]$ is approximately similar to $\operatorname{diag}\left(\lambda_{1}^{*}, \cdots, \lambda_{n}^{*}\right)$ [1].

Now as a consequence of the above results we have the following theorems and corollaries.

THEOREM 2.1. For every $B(t) \in G^{n}$ there exists a distinguished set of solutions for the system $\dot{x}=B(t) x$ such that the associated Perron transformation is almost periodic. 
Proof. If $B(t) \in G^{n}$ then $B(t)$ satisfies condition I and the result follows from Lemma 2.3.

TheOREM 2.2. If $B(t) \in F^{n}$ and satisfies condition I then $B(t) \in G^{n}$.

Proof. This is an immediate consequence of Lemmas 2.3 and 2.4.

CoROllary 2.1. Let $A(t) \in F^{n}$ be a symmetric matrix and assume that the constant terms of $A(t)$ form a matrix $A_{0}$ which has $n$ distinct real characteristic roots; then $A(t) \in G^{n}$.

Proof. Now

$$
\begin{aligned}
\exp \left[\int_{0}^{t} A(s) d s\right] & =\exp \left(\left[A_{0}+(1 / t) \int_{0}^{t}\left(A(s)-A_{0}\right) d s\right] t\right) \\
& \left.=\exp \left(A_{0} t\right) \cdot \exp \left(\left[(1 / t) \int_{0}^{t} A(s)-A_{0}\right) d s\right] t\right)
\end{aligned}
$$

is a fundamental solution for our system. However, given any $\mu>0$ there exists an $M$ such that

$$
(1 / M) \exp (-\mu|t|) \leqq \exp \left(\int_{0}^{t}\left(A(s)-A_{0}\right) d s\right) \leqq M \exp (\mu|t|)
$$

due to the fact that $A(s)$ is almost periodic. Since $A_{0}$ has $n$ distinct roots $\lambda_{1}, \cdots, \lambda_{n}$ it is clear that by forming appropriate linear combinations of the columns of $\exp \left[\int_{0}^{t} A(s) d s\right]$ we may obtain the fact that $A(t)$ satisfies condition I. Corollary 2.1 now follows from Theorem 2.2.

THEOREM 2.3. The set $G^{n}$ is open in the space $F^{n}$.

Proof. Lemma 2.1 states that if $B(t) \in G^{n}$ then there is an open set in $M_{n}$ containing $B(t)$ such that every element in this open set satisfies condition I. If we consider the restriction of this open set to $F^{n}$ our result now follows from Theorem 2.2.

3. Counterexample. The preceding results show us that the set of matrices in $F^{n}$ for which there exists a Perron transformation which is almost periodic contains an open set. We now observe that for $n \geqq 2$ this set is not itself open. The following example establishes this fact and demonstrates that the distinctness of the $\lambda_{i}$ is essential in condition I. For in the example that follows the inequalities of condition I hold with $\lambda_{1}=\lambda_{2}=\mu=0$ and the constant $M$ suitably chosen, yet there exist no almost periodic Perron transformations for $\mu \neq 0$. The example is for the case $n=2$, however, it is clear that a similar construction can be made for any $n>2$. 
EXAMPLE 3.1. Let $f(t)$ be an almost periodic function whose mean is zero but whose integral is not almost periodic. Then consider the system

$$
\left(\begin{array}{l}
\dot{x} \\
\dot{y}
\end{array}\right)=\left(\begin{array}{cc}
0 & \mu f(t) \\
-\mu f(t) & 0
\end{array}\right)\left(\begin{array}{l}
x \\
y
\end{array}\right) .
$$

Now for $\mu=0$ there clearly exists a Perron transformation which is almost periodic, in fact, all possible Perron transformations are given by constant matrices and so are almost periodic. For $\mu \neq 0$, the vectors

$$
v_{1}=\left(\begin{array}{c}
\sin \left(\int_{0}^{t} \mu f(s) d s\right) \\
\cos \left(\int_{0}^{t} \mu f(s) d s\right)
\end{array}\right) \text { and } v_{2}=\left(\begin{array}{r}
\cos \left(\int_{0}^{t} \mu f(s) d s\right) \\
-\sin \left(\int_{0}^{t} \mu f(s) d s\right)
\end{array}\right)
$$

form a basis for the solution space. We now show that for any solution $\rho(t)=a v_{1}+b v_{2}$ the vector $\rho(t) /\|\rho(t)\|$ is not almost periodic. This will show us that all possible Perron transformations fail to be almost periodic. Since we are interested in $\rho(t) /\|\rho(t)\|$ we may consider $\rho(t)=\left(a /\left[a^{2}+b^{2}\right]^{1 / 2}\right) v_{1}+\left(b /\left[a^{2}+b^{2}\right]^{1 / 2}\right) v_{2}$ or rewriting we have

$$
\begin{aligned}
\rho(t) & =\left[\begin{array}{l}
\sin \theta \sin \left(\int_{0}^{t} \mu f(s) d s\right)+\cos \theta \cos \left(\int_{0}^{t} \mu f(s) d s\right) \\
\sin \theta \cos \left(\int_{0}^{t} \mu f(s) d s\right)-\cos \theta \sin \left(\int_{0}^{t} \mu f(s) d s\right)
\end{array}\right] \\
& =\left(\begin{array}{l}
\cos \left(-\theta+\int_{0}^{t} \mu f(s) d s\right) \\
\sin \left(-\theta+\int_{0}^{t} \mu f(s) d s\right)
\end{array}\right) .
\end{aligned}
$$

Since $\|\rho(t)\|=1$, we have that $\rho(t) /\|\rho(t)\|=\rho(t)$ which is not almost periodic. This completes our example.

We note finally that in the case of an almost periodic matrix $A(t)$ the inequalities in condition I may be weakened so that one only assumes that they are satisfied for $t$ and $t_{0}$ in some half line. Then by passing to an element $A^{*}(t)$ in the hull of $A(t)$ one can establish that this matrix satisfies condition I. But by Lemma 2.2 the result then follows for all the matrices in the hull of $A^{*}(t)$ and so in particular for $A(t)$. We should note, however, that the original set of solutions satisfying the weakened inequalities need not be a distinguished set. 


\title{
BIBLIOGRAPHY
}

1. J. Lillo, Continuous matrices and the stability theory of differential systems, Math. Z. vol. 73 (1960) pp. 45-58. 138.

2. —-, Perturbations of nonlinear systems, Acta Math. vol. 103 (1960) pp. 123-

3. - Linear differential equations with almost periodic coefficients, Amer. J. Math. vol. 81 (1959) pp. 37-45.

4. L. Markus, Continuous matrices and the stability of differential systems, Math. Z. vol. 62 (1955) pp. 310-319.

5. W. T. Reid, Remarks on a matrix transformation for linear differential equations, Proc. Amer. Math. Soc. vol. 8 (1957) pp. 708-712.

RIAS

\section{METRIC TERNARY DISTRIBUTIVE SEMI-LATTICES}

\author{
S. P. AVANN
}

In this paper we show that the ternary operation of a metric ternary distributive semi-lattice, a generalization of the ternary Boolean algebra of Grau [2], uniquely minimizes ternary distance. This generalizes a result of Birkhoff and Kiss [1, Corollary 1, p. 749]. We show, conversely, that in a metric space unique minimizing of ternary distance determines a ternary operation with respect to which the space is a ternary distributive semi-lattice. Particularly, a lattice whose graph satisfies the unique minimal ternary distance condition and certain finiteness conditions must be distributive. This answers a question proposed by Birkhoff and Kiss [1, p. 750].

1. Definitions and postulates. We state our results at the close of this section.

A ternary distributive semi-lattice, hereinafter abbreviated TDSL, is a set of $J$ elements closed with respect to a ternary operation $(a, b, c)$ satisfying the following identities.

(T1) $(a, a, b)=a$.

(T2) $(a, b, c)$ is invariant under all 6 permutations.

(T3) $(a,(b, c, d), e)=((a, b, e), c,(a, d, e))$.

REMARK. The term, introduced by the author (Abstract 86, Bull. Amer. Math. Soc. vol. 54 (1948) p. 79), is a natural one in view of Lemma 3 . If in Lemma 3 there exists $a^{\prime} \in J$ satisfying

Received by the editors April 11, 1960 and, in revised form, July 18, 1960. 